\title{
Successful Treatment of Recurrent Hyperpyrexia, Stroke, Intracardiac Thrombosis and Inflammatory Pseudotumor Secondary to Behçet's Syndrome With Drug Therapy Alone
}

\section{Shuolin Wang}

Zhengzhou University https://orcid.org/0000-0001-8557-9186

\section{Lei Zhang}

Zhengzhou University First Affiliated Hospital

Tian-fang Li

Zhengzhou University First Affiliated Hospital

\section{Xu Liang}

Zhengzhou University First Affiliated Hospital

\section{Yajing Gao}

Zhengzhou University

Shengyun Liu ( $\nabla$ fccliusy2@zzu.edu.cn )

Zhengzhou University First Affiliated Hospital

\section{Case report}

Keywords: Behcet Syndrome, Cerebral Infarction, Heart, Inflammation, Thrombosis

Posted Date: April 13th, 2021

DOI: https://doi.org/10.21203/rs.3.rs-403897/v1

License: (c) (i) This work is licensed under a Creative Commons Attribution 4.0 International License.

Read Full License 


\section{Abstract}

\section{Background}

Behçet's syndrome (BS) is an inflammatory vessel disease characterized by recurrent oral and genital ulcers. It is a rare disorder that affects blood vessels throughout the body. It can affect any bodily system, including the skin, joints and cardiovascular, respiratory, central nervous and degestive systems. But cerebral infarction related to cerebral artery, intracardiac thrombosis (ICT) and inflammatory pseudotumor (IPT) are complications hard to see. And there is no effective therapy in clinic.

\section{Case presentation}

We report the case of a 17-year-old patient with a two-month history of recurrent hyperpyrexia without obvious infection symptoms. He had a medical history of cerebral infarction ten months ago. Antinuclear antibody (ANA), antiphospholipid antibodies, parasite antibodies and antineutrophil cytoplasmic antibodies (ANCA) were all negative, consequently. Echocardiography demonstrates heterogeneous echoes in the right ventricle and apex. He was suspected to have cardiac myxomas and infective endocarditis and underwent thoracotomy afterwards. Histological examinations showed the specimen is inflammatory mass of vascular origin and intracardiac thrombus (ICT). However, the hyperpyrexia and intracardiac masses relapsed in two months. The antibiotic treatment failed to control the temperature and he was diagnosed as BS and treated with prednisone, aspirin and oral cyclophosphamide. Since then, the body temperature turned to normal and the size of intracardiac mass maintained stable.

\section{Conclusion}

BS should be considered as a causative factor in young man with stroke, intracardiac masses and hyperpyrexia. Glucocorticoid and immunosuppressive agents may be effective for IPT and ICT in patient with BS.

\section{Background}

Behcet Syndrome (BS) is a multisystem inflammatory disease that affects blood vessels of any size[1]. Though it is well known that genetic and environmental factors are both involved in the development of BS, its exact etiology remains unknown. Given that BS involves an inflammatory process, inflammationmediated conditions such as thrombosis, inflammatory pseudotumor (IPT) and brain infarction are potential complications of BS. The mechanisms underlying the formation of thrombosis in BS are not entirely understood. It is plausible to postulate that endothelial damage and dysfunction caused by vascular inflammation may be involved in the thrombotic process[2]. Once formed, thrombotic masses could potentially migrate to the heart resulting in intracardiac thrombosis (ICT). However, ICT is an infrequent yet lethal manifestation of BS[3]. Inflammatory pseudotumor is a benign tumor and its etiology in patients with BS remains unknown. Brain infarction is a potential but rarely reported complication in patients with BS. To the best of our knowledge, all of the three complications mentioned 
above rarely occur in a patient with BS. We present the first report of these complications in a patient with BS.

\section{Case Presentation}

A 17-year-old male was admitted to our hospital with a two-month history of recurrent hyperpyrexia without obvious evidence of infection. His past medical history included a brain infarction 10 months ago, in which magnetic resonance angiography (MRA) demonstrated middle cerebral artery stenosis (Fig. 1). He was managed on prednisolone, warfarin and atorvastatin. Physical examination revealed skin erythema and recurrent oral ulcers. All laboratory results were normal, except for leukocytosis and increased erythrocyte sedimentation rate (ESR) and C-reactive protein (CRP) levels. Antinuclear antibody (ANA), antiphospholipid antibodies, parasite antibodies and antineutrophil cytoplasmic antibodies (ANCA) were all negative. Echocardiography demonstrated heterogeneous echoes in the right ventricle and apex (Fig. 2A). Based on these results, he was suspected of having cardiac myxomas and infective endocarditis, for which he underwent a thoracotomy. Histological examination of the intracardiac lesion showed proliferative granulation and fibrous tissue with hemorrhagic necrosis and infiltration with lymphocytes, neutrophils and eosinophils. Based on immunohistochemical testing, the results were positive for smooth muscle actin (SMA), CD34 (vascular), erythroblast transformation specific-related gene (ERG) protein (vascular origin), Clemson (arteriole wall origin) and Desman (arteriole wall origin). After the thoracotomy, the patient received anti-infective agents and supportive care and was discharged from the hospital in a stable condition. However, the hyperpyrexia relapsed in two months and he was readmitted. Further blood tests showed raised CRP levels $(34.08 \mathrm{mg} / \mathrm{L})$ and slightly elevated ESR (13.00 $\mathrm{mm} / \mathrm{h}$ ). Echocardiography demonstrated heterogeneous echoes in the heart cavity (Fig. 2B) and heart contrast-enhanced ultrasound (CEUS) suggested thrombus formation in the right ventricle and inflammatory changes in the right atrium (Fig. 2C). Following administration of levofloxacin intravenously at a dose of 0.4 gram per day for 7 days, this failed to control the pyrexia. He was, therefore, referred to the rheumatology department for further evaluation. Based on the International Criteria for Behçet's Disease (ICBD, 2006, revised in 2014), he was diagnosed with BS and treated with prednisone $60 \mathrm{mg} /$ day, aspirin $100 \mathrm{mg} /$ day and oral cyclophosphamide $100 \mathrm{mg} /$ day for 2 weeks. He responded to this treatment and was subsequently discharged. During the one-year follow-up period, prednisone was replaced with methylprednisolone, which was tapered to $4 \mathrm{mg}$ per day. He has had no flare-up of BS and the size of the intracardiac mass has remained the same (Fig. 2D).

\section{Discussion}

Cerebral infarction is a very rare occurrence in a young male with negative antiphospholipid antibody and normal coagulation function, but BS could increase the risk according to a Korean study which demonstrated that the risk of stroke [HR: $1.65,95 \% \mathrm{Cl}: 1.09-2.50]$ was significantly increased in patients with BS than those without BS[4]. So, it is highly possible that BS played a pivotal role in causing stroke in this patient. A multicenter study of cerebral venous thrombosis suggested that the superior sagittal 
sinuses and transverse sinuses are most frequently affected in patients with BS[5]. However, arteries are infrequently affected, and parenchymal lesions caused by cerebral artery involvement are even rarely reported[6, 7]. In our patient, cerebral arteries involvement doesn't manifest aneurysm or aneurysmal dilatation, but rather, more rarely, stenosis of middle cerebral artery. Although according to the latest European league against rheumatism (EULAR) recommendations, immunosuppressive agents should be used with high-dose glucocorticoids[8], the patient refused to take these immunosuppressants. Failure to administer immunosuppressive agents and the cessation of glucocorticoid after discharge may be the cause of his relapse.

Cardiac involvement in BS, especially IPT and ICT, are extremely rare. Therefore, they are often missed during the diagnostic workup and sometimes misdiagnosed as cardiac tumors, with eventual removal by surgery. Although surgical resection seems to be an effective therapeutic choice, it is inappropriate in patients with BS patients due to the risk of surgical complications and the development of calcified scars on the endocardium[3, 9]. Surgical resection is only undertaken when there are life-threatening symptoms.

To avoid unnecessary surgery, it is necessary to have a high index of suspicion for BS when intracardiac masses are found. Heart CEUS may improve the diagnostic accuracy of ICT[10]. In virtue of the rarity of ICT, CEUS is commonly used to identify hepatic IPT. However, it seemed to be quite useful in identifying intracardiac IPT in our case.

As presented in our case study, treatment with drugs is an alternative non-invasive treatment for ICT. Although there are no standard treatment protocols for ICT, azathioprine, cyclophosphamide or cyclosporine-A may be effective in patients with mild-to-moderate disease and cyclophosphamide suitable for patients with severe disease, according to the EULAR recommendation[8]. Though drug therapy alone was used in effectively treating our patient, further investigations are needed on effective drug therapies for IPT in patients with BS. Notably, although ERG does not differentiate between benign and malignant tumors, the sensitivities and specificities of ERG expression in vasogenic tumors are high $[11,12]$. So, we have shown for the first time that IPT may be directly caused by BS based on histological evidence and this implies that IPT is inflammatory and vasculogenic. The relationship between IPT and BS may provide a basis for understanding the underlying mechanisms of IPT formation and provide new opportunities for potential treatment.

\section{Conclusion}

In conclusion, this is the first report of a young male with BS who presented with all three complications of stroke, ICT and IPT and was successfully treated with drug therapy alone. For the first time, we have also provided a plausible underlying etiology for the relationship between BS and IPT and an alternative therapeutic approach.

\section{Abbreviations}


BS: Behçet's syndrome; ICT: Intracardiac thrombosis; IPT: Inflammatory pseudotumor; ESR: Erythrocyte sedimentation rate; CRP: C-reactive protein; ANA: Antinuclear antibody; ANCA: Antineutrophil cytoplasmic antibodies; SMA: Smooth muscle actin; ERG: Erythroblast transformation specific-related gene; CEUS: Contrast-enhanced ultrasound; EULAR: European league against rheumatism

\section{Declarations}

\section{Acknowledgements}

This study was supported by Science and Technology Development Program of Henan province (SB201901020) and Henan Province Medical Science and Technology Program Provincial and Ministerial Joint Projects (SBGJ202003024).

\section{Authors' contributions}

SL. Wang designed the study and wrote the manuscript. L. Zhang and X. Liang carried out laboratory tests and contributed to write the manuscript. TF. Li and YJ. Gao wrote and critically reviewed the manuscript. SY. Liu designed the study and critically reviewed the manuscript. All authors approved the final version of the manuscript.

\section{Funding}

No funding was obtained

\section{Availability of data and materials}

Data sharing is not applicable to this article as no datasets were generated or analysed during the current study.

\section{Declarations}

\section{Ethics approval and consent to participate}

This study was approved by the Ethical Committee of the First Affiliated Hospital of Zhengzhou University (Ethical approval number: 2020-KY-447).

\section{Consent for publication}

Consent was obtained from the patient

\section{Competing interests}

The authors declare that they have no competing interests

\section{Author details}


${ }^{1}$ Department of Rheumatology, Zhengzhou University First Affiliated Hospital, Zhengzhou, China

${ }^{2}$ Nursing and health school, Zhengzhou University, Zhengzhou, China

\section{References}

1. Sakane T, Takeno M, Suzuki N, Inaba G: Behçet disease. The New England Journal of Medicine 1999, 341:8.

2. Seyahi E: Behcet's disease: How to diagnose and treat vascular involvement.Best Pract Res Clin Rheumatol 2016, 30:279-295.

3. Aksu T, Tufekcioglu 0: Intracardiac thrombus in Behcet's disease: four new cases and a comprehensive literature review. Rheumatol Int 2015, 35:1269-1279.

4. Ahn H-S, Lee D, Lee SY, Kim YH, Lee JH, Yu DS, Han K-D, Park YG, Lee YB: Increased Cardiovascular Risk and All-cause Death in Patients with Behçet Disease: A Korean Nationwide Population-based Dynamic Cohort Study. The Journal of Rheumatology 2019.

5. Uluduz D, Midi I, Duman T, Colakoglu S, Tufekci A, Bakar M, Nazliel B, Tascilar N, Goksan B, Sungur $M A$, et al: Behcet's disease as a causative factor of cerebral venous sinus thrombosis: subgroup analysis of data from the VENOST study.Rheumatology (Oxford) 2018.

6. Saadoun D, Asli B, Wechsler B, Houman H, Geri G, Desseaux K, Piette J, Huong dL, Amoura Z, Salem $\mathrm{T}$, et al: Long-term outcome of arterial lesions in Behçet disease: a series of 101 patients.Medicine 2012, 91:18-24.

7. Desbois A, Wechsler B, Cluzel P, Helft G, Boutin D, Piette J, Cacoub P, Saadoun D: Cardiovascular involvement in Behçet's disease.La Revue de medecine interne 2014, 35:103-111.

8. Hatemi G, Christensen R, Bang D, Bodaghi B, Celik AF, Fortune F, Gaudric J, Gul A, Kotter I, Leccese P, et al: $\mathbf{2 0 1 8}$ update of the EULAR recommendations for the management of Behcet's syndrome.Ann Rheum Dis 2018, 77:808-818.

9. Seyahi E: Behçet's disease: How to diagnose and treat vascular involvement.Best practice \& research Clinical rheumatology 2016, 30:279-295.

10. Takeuchi M, Ogunyankin K, Pandian N, McCreery T, Sweitzer R, Caldwell V, Unger E, Avelar E, Sheahan $\mathrm{M}$, Connolly R: Enhanced visualization of intravascular and left atrial appendage thrombus with the use of a thrombus-targeting ultrasonographic contrast agent (MRX-408A1): In vivo experimental echocardiographic studies.J Am Soc Echocardiogr 1999, 12:1015-1021.

11. Patai B, Peterfy N, Szakacs N, Sapi Z, Hetthessy J: Papillary Endothelial Hyperplasia (Masson Tumor) of the Hand. Surgical and Pathological Consideration from Seven Cases Using New Vascular Markers. Pathology oncology research : POR 2020, 26:2083-2090.

12. Xu J, Hu S, Li S, Wang W, Zhou X, Wu Y, Su Z, Cheng X, Gao Y, Zheng Q: Laparoscopic resection of hepatic epithelioid hemangioendothelioma: report of eleven rare cases and literature review. World journal of surgical oncology 2020, 18:282. 
Figures

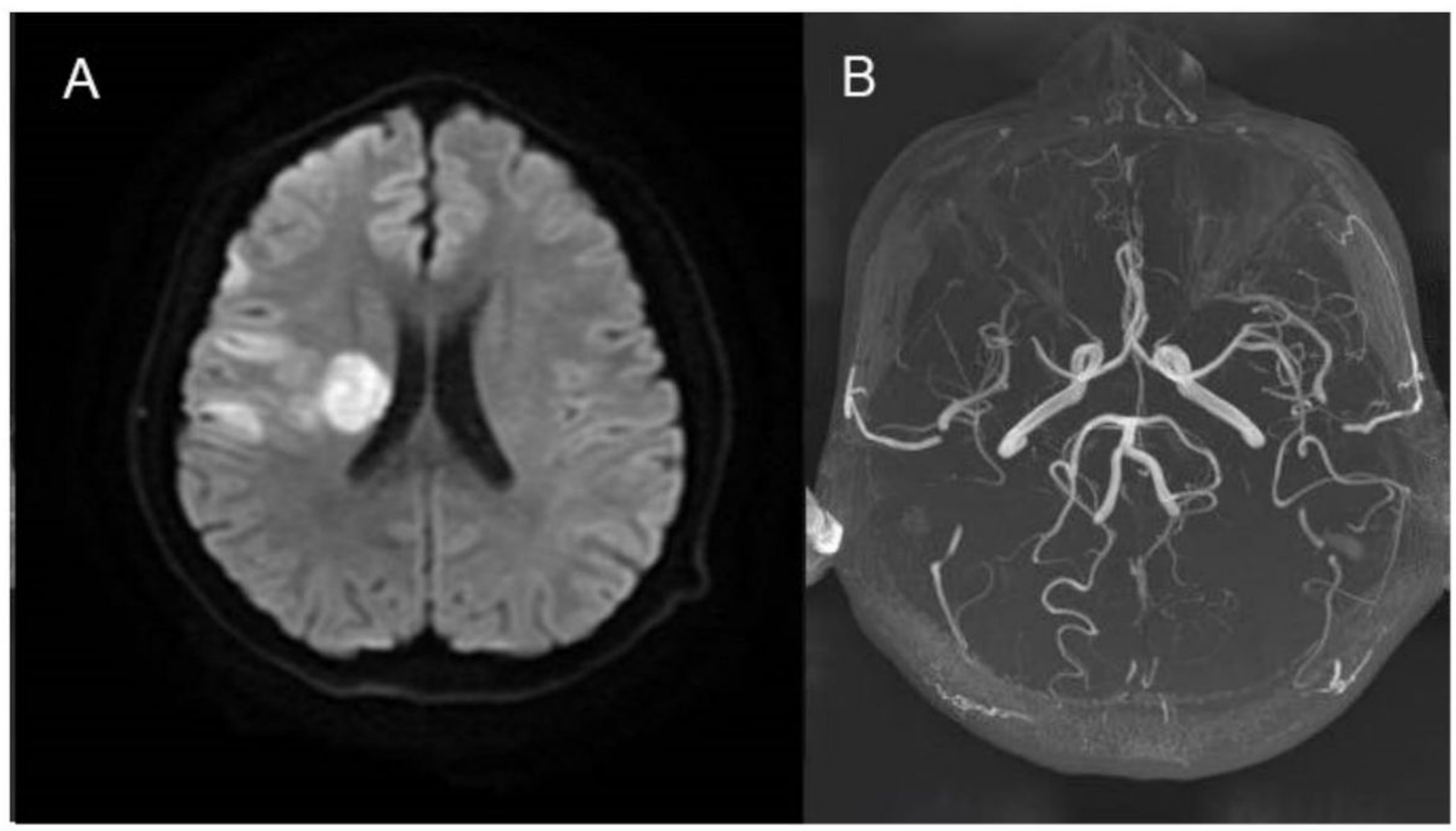

\section{Figure 1}

Finding on head magnetic resonance. A) Acute cerebral infarction of the right brain suggested by DWl; B) Stenosis of the right middle cerebral artery showed by MRA 


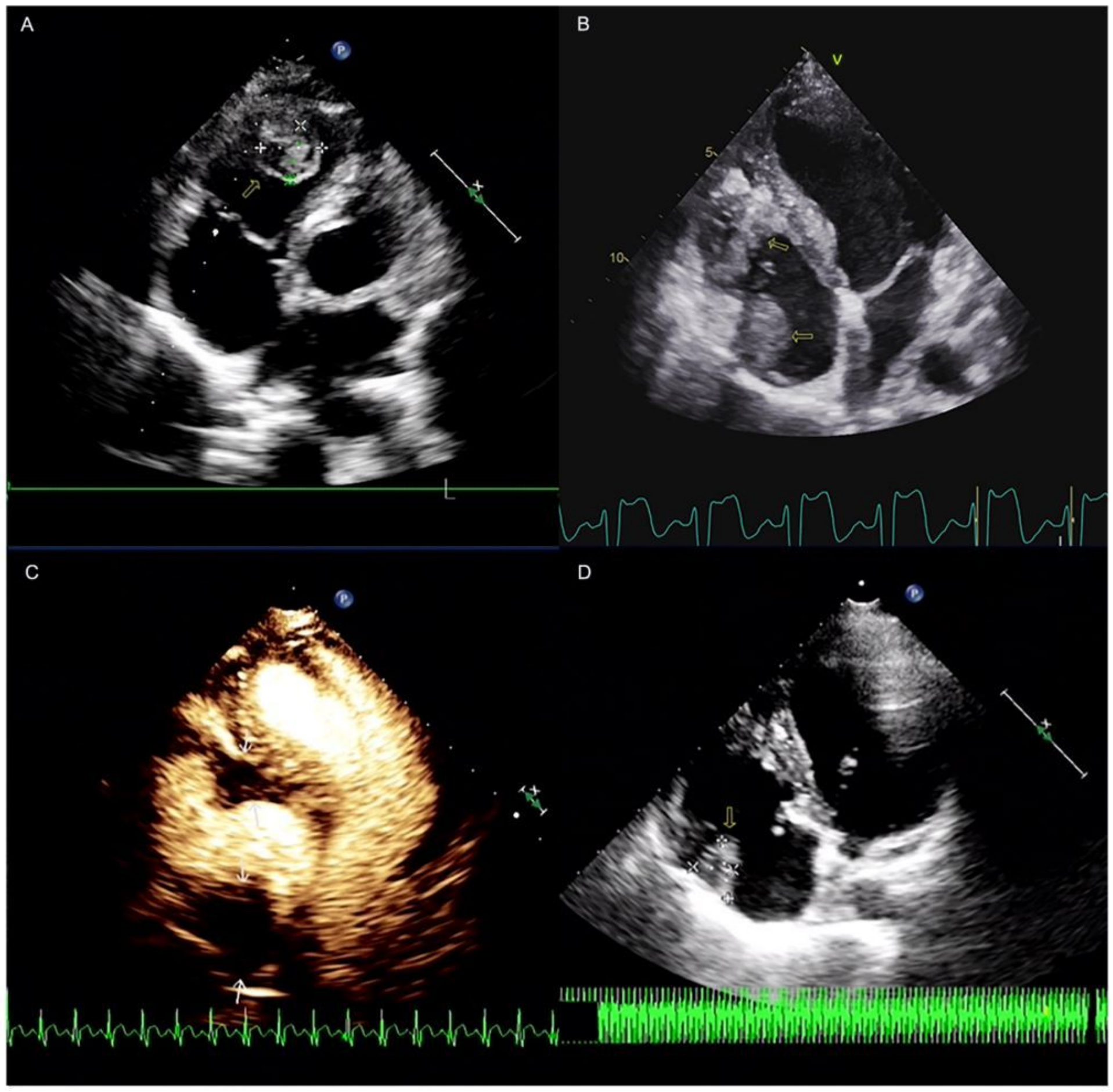

\section{Figure 2}

The development of the cardiac mass demonstrated by ultrasonography. A) Echocardiography demonstrated heterogeneous echoes in the right ventricle and apex at the time of onset $B$ ) Echocardiography demonstrated heterogeneous echoes in the right ventricle and atrium at the first relapse. C) Heart contrast-enhanced ultrasound showed hyperecho and contrast agent filling in the right ventriele (suggesting thrombus) and atrium (suggesting inflammatory changes) at the first relapse. D) Echocardiography showed the size of the intracardiac mass remained the same during the follow-up after two years. 